Published in final edited form as:

Exp Eye Res. 2010 February ; 90(2): 196. doi:10.1016/j.exer.2009.10.006.

\title{
Characteristics of Period Doubling in the Rat Cone Flicker ERG
}

\author{
Manthan R. Shah ${ }^{1}$, Kenneth R. Alexander ${ }^{1}$, Harris Ripps ${ }^{1,2,3,5}$, and Haohua Qian ${ }^{1,2,4}$ \\ ${ }^{1}$ Department of Ophthalmology and Visual Sciences, University of Illinois at Chicago, Chicago, \\ Illinois 60612, USA \\ 2Department of Physiology and Biophysics, University of Illinois at Chicago, Chicago, Illinois 60612, \\ USA \\ ${ }^{3}$ Department of Anatomy and Cell Biology, University of Illinois at Chicago, Chicago, Illinois 60612, \\ USA \\ ${ }^{4}$ Department of Biological Sciences, University of Illinois at Chicago, Chicago, Illinois 60612, USA \\ ${ }^{5}$ Marine Biological Laboratory, Woods Hole, MA 02543
}

\section{Abstract}

When the eye is stimulated by a flickering light, the electroretinogram (ERG) and other electrophysiological responses in the visual pathway often exhibit period doubling. This phenomenon is manifested as an alternation in the shape of the response waveform from cycle to cycle, and also as spectral components at the half-fundamental frequency $(\mathrm{F} / 2)$ and its odd multiples. Although period doubling has been described in humans as well as in other animals, its features in the rodent flicker ERG have not been characterized. We investigated the properties of period doubling in the rat cone flicker ERG elicited with full field, sinusoidal photic stimuli. Period doubling was observed when the temporal frequency of the stimulus was in the range of 20 to $30 \mathrm{~Hz}$. The F/2 component of the Fourier spectrum of the ERG was more pronounced than its odd harmonics. The magnitude of the cycle-to-cycle variation in amplitude differed depending on whether measurements were based on peak-to-trough or trough-to-peak amplitudes, owing to the relative phase relationship between F/ 2 and $\mathrm{F}$ as a function of stimulus frequency. The frequency-response characteristics of period doubling varied with stimulus contrast, such that reducing the contrast shifted the peak F/2 amplitude to a lower stimulus frequency. Period doubling was evident in rat eyes in which PDA was administered intravitreally, indicating that the phenomenon can occur independently of OFFpathway activity in the rat retina. The period doubling properties we observed in the flicker ERG response of the rat cone system provide constraints on the nature of the nonlinear feedback mechanism presumed to underlie the period doubling phenomenon.

\section{Keywords}

Electroretinogram; flicker ERG; rodent; period doubling; cone pathway; pharmacology; sinusoidal stimuli; temporal frequency

Corresponding Author: Dr. Haohua Qian, Department of Ophthalmology and Visual Sciences, University of Illinois at Chicago, 1855 W. Taylor Street, Chicago, IL 60612, Phone: 312-413-7347; Fax: 312-996-7773; hqian@uic.edu.

Publisher's Disclaimer: This is a PDF file of an unedited manuscript that has been accepted for publication. As a service to our customers we are providing this early version of the manuscript. The manuscript will undergo copyediting, typesetting, and review of the resulting proof before it is published in its final citable form. Please note that during the production process errors may be discovered which could affect the content, and all legal disclaimers that apply to the journal pertain. 


\section{Introduction}

The electroretinogram (ERG) elicited by a periodic photic stimulus is used frequently as an objective and non-invasive method for the assessment of retinal function. Under certain conditions, however, the waveform of the cone-derived ERG response to a flickering stimulus can exhibit alternations in amplitude and/or shape from cycle to cycle (Crevier and Meister, 1998; Alexander et al., 2005). This response alternation, termed period doubling, is reflected in the Fourier spectrum of the ERG waveform as components at half the fundamental frequency (F/2) and its odd multiples (Crevier and Meister, 1998). Period doubling has been observed in light-evoked responses recorded from various sites in the visual pathway in a number of species. For example, period doubling has been reported for flicker ERG recordings from human and salamander eyes (Crevier and Meister, 1998; Alexander et al., 2005), in the mass electrical potential of the optic nerve in salamander retina (Crevier and Meister, 1998), in single cell recordings from mouse retinal ganglion cells (Schwartz and Berry, 2008), and in the human visual evoked potential (VEP) (Crevier and Meister, 1998).

It has been proposed that period doubling reflects the operation of a nonlinear feedback signal with an exponential decay that alters response gain, in combination with electrical coupling between neural elements that leads to synchronous responses (Crevier and Meister, 1998). Pharmacological studies of period doubling in salamander retina have demonstrated that the phenomenon is not observed in the photoreceptor response, it is resistant to blockade of the retinal ON pathway, and it is absent when OFF retinal activity is inhibited (Crevier and Meister, 1998). This has led to the conclusion that the minimum circuit necessary to generate period doubling consists of cone photoreceptors and OFF bipolar cells (Crevier and Meister, 1998).

In a previous study, we examined the temporal response properties of the rat cone flicker ERG elicited with stimulus frequencies of 2 to $30 \mathrm{~Hz}$ (Qian et al., 2008). We noticed that, when stimulus frequency was in the range of 20 to $30 \mathrm{~Hz}$, the amplitude spectrum of a number of the recordings contained a prominent $F / 2$ component. The purpose of the present study was to examine this phenomenon more closely, focusing on the frequency range of 20 to $30 \mathrm{~Hz}$ and using frequency steps of approximately $1 \mathrm{~Hz}$. In addition to measurements of the F/2 response component, we also examined ERG response amplitude, which was quantified in two ways: (1) peak-to-trough (P-T) amplitude and (2) trough-to-peak (T-P) amplitude.

The contribution of the retinal OFF pathway to period doubling in the rat flicker ERG was determined by examining the effect of intravitreal administration of cis-2,3-piperidinedicarboxylic acid (PDA), a specific inhibitor of OFF-pathway activity. In comparison to the ERG of salamander, which reflects the activity of both ON and OFF pathways, the rodent flicker ERG is dominated by retinal ON-pathway activity, such that blocking the ON pathway almost completely suppresses the ERG response (Krishna et al., 2002; Qian et al., 2008). Therefore, while it is possible to examine period doubling in the rat eye when the retinal OFF pathway is blocked, it is difficult to quantify period doubling when the ON pathway is blocked, due to the small amplitude of the remaining ERG response.

\section{Materials and Methods}

\section{Animals}

Adult rats (both sexes, weight 250-500 g) of both albino (Sprague Dawley) and pigmented (Long Evans) strains were used for this study. No systematic differences were observed in the flicker ERG recordings from these two strains, and so the data from both were pooled. For each rat, the ERGs from both eyes were recorded sequentially. All experimental procedures conformed to the statement on animal care of the Association for Research in Vision and 
Ophthalmology, and adhered to the guidelines for the Care and Use of Laboratory Animals formulated by the Animal Care Committee of the University of Illinois at Chicago.

\section{ERG recordings}

The instrumentation and recording procedures were described in our previous publication (Qian et al., 2008). Briefly, rats were anesthetized with ketamine and xylazine, and their pupils were dilated with topical phenylephrine and tropicamide. Body temperature was maintained at $\sim 37^{\circ} \mathrm{C}$ with a heating pad. After applying a topical anesthetic (proparacaine, $0.5 \%$ ), ERG responses were recorded from a chlorided silver wire electrode placed in the center of the cornea and connected to the input stage of a Grass AC amplifier (Model P511, bandwidth 0.3 to $300 \mathrm{~Hz}$ without a $60 \mathrm{~Hz}$ notch filter; sampling frequency, $2 \mathrm{kHz}$ ).

\section{Light Stimulation}

The stimulus luminance was modulated sinusoidally at temporal frequencies ranging from 20 to $30 \mathrm{~Hz}$, with a mean luminance of $100 \mathrm{~cd} / \mathrm{m}^{2}$. At this luminance level, rat rods are saturated (Xu et al., 2003), and ERG responses reflect the activity of cone pathways in the retina. The stimulus contrast was defined as $\left(L_{\max }-L_{\min }\right) /\left(L_{\max }+L_{\min }\right)$, where $L_{\max }$ and $L_{\min }$ are the maximum and minimum stimulus luminances, respectively. In most experiments, the stimulus contrast was $90 \%$. For experiments in which we specifically examined the effect of contrast, flicker ERGs were also measured at stimulus contrasts of $30 \%$ and $60 \%$.

Following exposure to room illumination, the rat eye was adapted to the stimulus mean luminance for approximately 2 min prior to an ERG recording. Two stimulus protocols were used, as illustrated in Fig. 1. Protocol 1 (Fig. 1, top) was adopted from our previous study (Qian et al., 2008), in which each stimulus, containing an even number of cycles, was presented for approximately 10 seconds. Recordings with this protocol, which was used primarily for studying the spectral components of the flicker ERG waveform, were obtained from 36 rats (72 eyes). Protocol 2 (Fig. 1, bottom) consisted of 12 bursts of sinusoidally modulated light, each with a duration of approximately $1 \mathrm{~s}$ and containing an even number of cycles, with an inter-burst interval of $0.4 \mathrm{~s}$. Recordings were obtained from 20 rat eyes using this protocol. This second protocol was used primarily in the evaluation of cycle amplitudes over time, although spectral analysis was also performed on these recordings. For both protocols, ERG recordings were synchronized with the stimulus onset.

\section{Intravitreal injection}

PDA was delivered to the anesthetized eye by intravitreal injection. A glass capillary needle was introduced into the vitreous cavity by piercing the sclera $3 \mathrm{~mm}$ posterior to the temporal limbus at approximately a 45-degree angle to the optical axis. The injection site was monitored under a dissecting microscope, and a $3-\mu \mathrm{l}$ aliquot of a solution containing $100 \mathrm{mM}$ of PDA was injected into each eye. The final vitreal concentration $(\sim 8 \mathrm{mM})$ was derived by assuming complete mixing in the rat vitreous with an estimated volume of $38 \mu \mathrm{l}$ (Xu et al., 2003). ERG measurements were made following PDA injection. There was no ERG measurement prior the PDA injection in order to minimize the time under anesthesia.

\section{Data Analysis}

ERG amplitude spectra were derived from discrete Fourier transforms using the Matlab Signal Processing Toolbox (The Mathworks, Boston, MA). The amplitudes of the individual responses of the ERG waveforms were measured as both P-T and T-P amplitudes. The mean amplitudes of the responses to the odd and even stimulus cycles were then obtained at each stimulus frequency. For ERG recordings obtained with Protocol 1, about $500 \mathrm{~ms}$ of data at the beginning and end of the recording were omitted. The exact length that was omitted depended 
on the stimulus period and was an even number of cycles. As a result, spectral analyses of ERGs obtained with Protocol 1 were based on approximately 9-s segments of continuous data, consisting of an even number of cycles. For recordings obtained with Protocol 2, the ERG responses to the twelve 1-s stimulus bursts were averaged, after confirming that the characteristics of period doubling were similar for each burst response. Amplitude measures and spectral analyses were based on the $5^{\text {th }}$ through the $18^{\text {th }}$ response cycles of the averaged waveforms obtained with Protocol 2. As a criterion for period doubling, we adopted a rule of an $\mathrm{F} / 2$ response that was greater than 3 times the noise level at neighboring frequencies (which exceeds the 5\% significance level; Meigen and Bach, 1999) for at least one stimulus frequency in the range of 20 to $30 \mathrm{~Hz}$. If this criterion was met for an individual eye, then the ERG responses at all stimulus frequencies for that eye were included in the spectral and amplitude analyses.

\section{Results}

\section{Characteristics of period doubling}

Fig. 2 illustrates representative ERG waveforms elicited from a rat eye at a range of stimulus frequencies using Protocol 1, together with the stimulus waveform (bottom trace). For clarity of display, the ERG waveforms are plotted with respect to stimulus period rather than time. Each trace represents the 9-s middle portion of each ERG waveform, which was divided into 8 -cycle segments and then averaged for the plots. An alternation in response waveform shape from cycle to cycle is apparent for stimulus frequencies ranging from 22.7 to $27.8 \mathrm{~Hz}$.

The amplitude spectrum of the entire 9-s ERG response at $23.8 \mathrm{~Hz}$ (the stimulus frequency at which there was the maximum degree of period doubling) is plotted in Fig. 3. As reported previously (Qian et al., 2008), the amplitude spectrum of the rat ERG is dominated by the fundamental response $(\mathrm{F})$, with a substantial contribution from the second harmonic (2F). In addition, there was a prominent $\mathrm{F} / 2$ component, indicative of period doubling. Additional odd multiples of $\mathrm{F} / 2$ (i.e., $3 \mathrm{~F} / 2$ and $5 \mathrm{~F} / 2$ ) were also present, although they were relatively small in amplitude. Of note, the responses at the even multiples of $\mathrm{F} / 2$ cannot be distinguished from the responses to the fundamental and its harmonics. The amplitudes of the F/2 components of all of the ERG waveforms of Fig. 2 are plotted in Fig. 4A. The amplitudes of F/2 followed a bell-shaped curve as a function of stimulus frequency, with the maximum amplitude at a stimulus frequency of $23.8 \mathrm{~Hz}$.

We observed substantial variation in the amplitude of $\mathrm{F} / 2$ from one rat to the next, as well as between the two eyes of the same animal. By our criterion for period doubling, sixteen of the 36 rats tested (44.4\%) showed period doubling in both eyes, nine (25\%) exhibited period doubling in only one eye, and eleven (30.6\%) showed no period doubling in either eye. Therefore, of the 72 albino and pigmented rat eyes tested, 41 eyes (57\%) exhibited some degree of period doubling in the flicker ERG.

Fig. 4B plots the relationship between stimulus frequency and the mean amplitude of the F/2 component of the ERG recordings from the 41 rat eyes that showed period doubling by our criterion. This function had the same overall shape as the curve in Fig. 4A but was much flatter, due in part to individual variations in the stimulus frequency at which the maximum amplitude of F/2 occurred. This stimulus frequency had a mean and standard deviation of $25.3 \pm 0.34 \mathrm{~Hz}$. Relative to the amplitude of $\mathrm{F}$, the amplitude of $\mathrm{F} / 2$ at its maximum was $0.51 \pm 0.06$ and the amplitude of $3 \mathrm{~F} / 2$ was only $0.11 \pm 0.01$.

Fig. 5 presents examples of waveforms recorded from a representative rat eye across a range of stimulus frequencies using Protocol 2. In this figure, each waveform is the average of the responses to the twelve 1-s stimulus bursts. Even though the eye was adapted to the mean 
luminance level, large transients were observed at flicker onset and offset at every frequency. The overall response amplitude decreased with increasing stimulus frequency, as observed previously in the rat cone flicker ERG (Qian et al., 2008). For the $20 \mathrm{~Hz}$ stimulus, there was little variation in the response amplitude across cycles. Period doubling, manifested as an alternation in the response waveform from cycle to cycle, was evident for this rat eye at stimulus frequencies from 21.7 to $26.3 \mathrm{~Hz}$.

We measured the P-T and T-P amplitudes of the individual cycles of the flicker ERG from the 5th through the 18th response cycle (indicated by the arrows above each trace in Fig. 5). The amplitudes on the odd and even response cycles were averaged separately. The mean P-T and T-P amplitudes on odd and even cycles are plotted in Figs. 6A and 6B, respectively. It is apparent that the response amplitudes for the odd and even cycles differed depending on whether the measurements were of P-T or T-P amplitude. For P-T amplitudes (Fig. 6A), there were differences between the odd and even cycles at stimulus frequencies between approximately 21 and $25 \mathrm{~Hz}$, whereas for T-P amplitudes, the differences occurred at frequencies between approximately 23 and $27 \mathrm{~Hz}$. The dissimilarity between these two amplitude measures is further evident in Fig. 6C, which plots the amplitude differences between odd and even cycles for both metrics.

Fig. 7 plots the mean differences between the amplitudes of the odd and even response cycles for both the P-T (triangles) and T-P (diamonds) measures for the 8 of 20 rat eyes that showed period doubling using Protocol 2. The function for P-T amplitude differences (triangles) showed a peak at lower stimulus frequencies than did the function for T-P amplitude differences (diamonds). Also shown in Fig. 7 are the mean amplitudes of the F/2 component (half-filled circles) of the same sections of the ERG waveforms that were used for the T-P and P-T amplitude measurements. The F/2 component exhibited a broader frequency-response function than that for either P-T or T-P amplitudes, and, in fact, conformed to the envelope of the amplitude difference functions.

The asymmetry observed between the two amplitude measures is due primarily to the relative phase relationship between F/2 and F. This is illustrated in Fig. 8, which demonstrates the effect of varying the phase of $\mathrm{F} / 2$ relative to $\mathrm{F}$ at a temporal frequency of $23.8 \mathrm{~Hz}$. Fig. $8 \mathrm{~A}$ presents three schematic waveforms that each represents the sum of F/2 and F components. For the middle waveform, the amplitudes and phases of $F / 2$ and $F$ correspond to those derived by spectral analysis from the 23.8-Hz ERG waveform shown in Fig. 5. The other two schematic waveforms in Fig. 8A represent the result of phase-shifting the F/2 component by $30 \mathrm{deg}$ in either direction relative to the phase of $\mathrm{F} / 2$ in the middle waveform. It is apparent that phaseshifting $F / 2$ relative to $F$ results in asymmetric waveform shapes that bear a similarity to the ERG waveforms plotted in Fig. 5. Of note, the phase shifts are plotted as positive to negative because the phases of the spectral components of the ERG waveforms become more negative with increasing stimulus frequency.

Fig. 8B plots the difference between even and odd cycles based on P-T (triangles) and T-P (diamonds) amplitudes as the phase of $F / 2$ was shifted relative to $F$. With a positive phase shift (such as occurs at lower stimulus frequencies), the amplitude difference was greater using the $\mathrm{P}-\mathrm{T}$ amplitude measure, as can also be seen by inspecting the top waveform in Fig. 8A. With a negative phase shift, the amplitude difference was greater using the T-P measure. The amplitude difference was minimized when the relative phase of $\mathrm{F} / 2$ corresponded to that of the actual 23.8-Hz waveform shown in Fig. 5. Thus, the phase relationship between F/2 and F is a major determinant of the asymmetry between the P-T and T-P amplitude measures. 


\section{Effect of contrast on period doubling}

The effect of stimulus contrast on period doubling is illustrated for one rat eye in Fig. 9A, which plots the amplitude of F/2 at each of three contrast levels using Protocol 1. It is apparent from this figure that the overall amplitude of the F/2 component increased with increasing stimulus contrast, and the stimulus frequency at which $\mathrm{F} / 2$ reached its peak amplitude shifted to a higher stimulus frequency with increasing contrast. The effect of stimulus contrast was examined in a total of 24 eyes (12 rats), of which 9 eyes exhibited period doubling. Fig. 9B plots the mean stimulus frequency at which the maximum amplitude of period doubling occurred in recordings from these 9 eyes at stimulus contrasts of $30 \%, 60 \%$, and $90 \%$. The peak of the $\mathrm{F} / 2$ amplitude function increased by 1.8 cycles for each $30 \%$ increment in contrast.

\section{Effect of PDA on period doubling}

Fig. 10A illustrates the relationship between F/2 amplitude and stimulus frequency for ERG recordings from a rat eye injected intravitreally with $8 \mathrm{mM}$ PDA, using a stimulus contrast of $90 \%$. The amplitude of the $\mathrm{F} / 2$ component was above the noise level at temporal frequencies below $24 \mathrm{~Hz}$, with a peak at approximately $21 \mathrm{~Hz}$. This amplitude function is quite different from that obtained in response to a stimulus of 90\% contrast in the absence of PDA (Fig. 9A), and in fact is more similar to that obtained at a stimulus contrast of $30 \%$. Of the 20 rat eyes injected with PDA, 12 gave F/2 responses having amplitudes that were 3 -fold greater than the noise level. Fig. 10B plots the mean F/2 amplitudes from these 12 rat eyes (triangles), together with the mean F/2 amplitudes of a group of 41 control rat eyes replotted from Fig. 4B (circles). PDA did not eliminate period doubling, but the peak stimulus frequency at which period doubling occurred was lower than for the control eyes.

\section{Discussion}

In the rat flicker ERG, period doubling was seen in response to photic stimuli with temporal frequencies in the range of 20 to $30 \mathrm{~Hz}$ (Fig. 2). In comparison, period doubling was observed in the flicker ERG of salamander retina at stimulus frequencies in the range of 10 to $20 \mathrm{~Hz}$ (Crevier and Meister, 1998), and period doubling in the human flicker ERG occurs at frequencies from approximately 30 to as high as $100 \mathrm{~Hz}$ (Alexander et al., 2008). It is unlikely that these differences are caused by the anesthetic used in this study. In a preliminary study in which we examined period doubling in rabbits anesthetized with the same agent, the ERGs showed period doubling in response to stimuli in the frequency range of 30 to $50 \mathrm{~Hz}$, with a strong $3 \mathrm{~F} / 2$ component (data not shown), which is similar to results observed in humans (Alexander et al., 2008). According to the model of period doubling proposed by Crevier and Meister (1998), the time constant of the feedback signal plays an important role in determining the temporal frequency at which period doubling occurs. Thus, the observed species differences in the temporal frequency range of period doubling may reflect differences in the kinetics of retinal signal processing.

Only about $50 \%$ of the rat eyes that were tested exhibited period doubling by our criterion (value of $\mathrm{F}$ that exceeded 3 times the noise level for at least one stimulus frequency). Of the eyes that showed period doubling, there was a large variation in both the peak amplitude and in the stimulus frequency at which period doubling reached its peak amplitude. Intersubject variability in the expression of period doubling in the flicker ERG has also been observed in human subjects (Alexander et al., 2005). The explanation for this variability is not entirely clear, but there are at least two factors that could be involved. First, it is possible that not all of a given class of retinal neurons might exhibit period doubling. For example, in a recent study utilizing multi-electrode array recordings of mouse ganglion cell responses, it was reported that only a small proportion (about $25 \%$ ) of these cells exhibited period doubling (Schwartz and Berry, 2008). Second, as discussed by Crevier and Meister (1998), the synchronous activity 
of a population of retinal cells is required to achieve a detectable level of period doubling in ERG recordings. Without such large-scale synchronization, the period-doubled responses of individual retinal neurons might tend to cancel in the full-field ERG.

The two methods of measuring amplitude that we used (P-T and T-P) produced different estimates of the frequency range over which period doubling occurred (Figs. 6 and 7). An asymmetry between P-T and T-P measurements can also be observed in ERG recordings from salamander retina (Crevier and Meister, 1998). As illustrated in Fig. 8, the asymmetry between $\mathrm{P}-\mathrm{T}$ and T-P amplitude measures can be attributed to a change in the relative phase relationship between F/2 and $\mathrm{F}$ across temporal frequency. It is apparent from Fig. 7 that the amplitude of $\mathrm{F} / 2$ as derived from a spectral analysis of the rat flicker ERG provides a more appropriate measure of period doubling than does the measurement of waveform amplitude.

We observed a shift of period doubling to higher stimulus frequencies with increasing stimulus contrast in rat (Fig. 9). In comparison, period doubling was displaced to lower stimulus frequencies with increasing contrast in the human flicker ERG (Alexander and Raghuram, 2007). The explanation for the different effect of stimulus contrast on the rat and human flicker ERG requires further investigation.

In salamander retina, period doubling was absent in the photoreceptor response and did not require inhibitory feedback among neurons or the integrity of the retinal ON pathway (Crevier and Meister, 1998). On the other hand, period doubling in salamander disappeared when the retinal OFF pathway was inhibited. However, we found that period doubling in the rat ERG could still be observed when retinal OFF-pathway activity was reduced by the intravitreal injection of PDA (Fig. 10). However, the frequency range of period doubling was displaced to lower stimulus frequencies following PDA injection, as if the stimulus were of a lower effective contrast. Of interest, it has been shown that the contrast sensitivity of the visual pathway depends on the integrity of both the retinal ON and OFF pathways (Schiller et al., 1986; Dolan and Schiller, 1994). It is possible, therefore, that suppressing the OFF pathway of the rat retina with an intravitreal injection of PDA reduces the overall contrast sensitivity of the visual system, and leads, in turn, to the shift of period doubling to a lower stimulus frequency range. However, PDA did not result in the reduction in the amplitude of $F$ that would be expected if the stimulus were of a lower effective contrast. This result indicates that the effect of PDA on period doubling did not simply reflect a general attenuation of the ERG response.

An explanation for the differential effect of PDA on period doubling in salamander and rat retinas is not readily apparent, but we note that the rat flicker ERG is dominated by the retinal ON pathway (Qian et al., 2008), whereas the salamander flicker ERG reflects the activity of both ON and OFF pathways, with a relatively greater contribution from the OFF pathway (Crevier and Meister, 1998). In view of the fact that period doubling in the rat flicker ERG can be generated independent of the retinal OFF pathway, it is reasonable to assume that period doubling is mediated by neurons in whichever pathway is dominant.

\section{Acknowledgments}

The authors thank Ms. Tara Nguyen for excellent technical support. This work was supported by a grant from the Pearle Vision Foundation (HQ), the Joyce Schroeder Fund (HQ), NIH research grant EY08301 (KRA), NIH core grant EY01792, an Alcon Research Institute Award (HR), RPB Senior Scientific Investigator Awards (KRA, HR), and an unrestricted departmental award from Research to Prevent Blindness, Inc.

\section{References}

Alexander KR, Raghuram A. Effect of contrast on the frequency response of synchronous period doubling. Vision Res 2007;47:555-563. [PubMed: 17074380] 
Alexander KR, Levine MW, Super BJ. Characteristics of period doubling in the human cone flicker electroretinogram. Vis Neurosci 2005;22:817-824. [PubMed: 16469190]

Alexander KR, Raghuram A, McAnany JJ. Comparison of spectral measures of period doubling in the cone flicker electroretinogram. Doc Ophthalmol 2008;117:197-203. [PubMed: 18357480]

Crevier DW, Meister M. Synchronous period-doubling in flicker vision of salamander and man. J Neurophysiol 1998;79:1869-1878. [PubMed: 9535954]

Dolan RP, Schiller PH. Effects of ON channel blockade with 2-amino-4-phosphonobutyrate (APB) on brightness and contrast perception in monkeys. Vis Neurosci 1994;11:23-32. [PubMed: 8011580]

Krishna VR, Alexander KR, Peachey NS. Temporal properties of the mouse cone electroretinogram. J Neurophysiol 2002;87:42-48. [PubMed: 11784728]

Meigen T, Bach M. On the statistical significance of electrophysiological steady-state responses. Doc Ophthalmol 1999;98:207-232. [PubMed: 10945442]

Qian H, Shah MR, Alexander KR, Ripps H. Two distinct processes are evident in rat cone flicker ERG responses at low and high temporal frequencies. Exp Eye Res 2008;87:71-75. [PubMed: 18555992]

Schiller PH, Sandell JH, Maunsell JH. Functions of the ON and OFF channels of the visual system. Nature 1986;322:824-825. [PubMed: 3748169]

Schwartz G, Berry MJ 2nd. Sophisticated temporal pattern recognition in retinal ganglion cells. J Neurophysiol 2008;99:1787-1798. [PubMed: 18272878]

Xu L, Ball SL, Alexander KR, Peachey NS. Pharmacological analysis of the rat cone electroretinogram. Vis Neurosci 2003;20:297-306. [PubMed: 14570251] 


\section{Protocol 1}

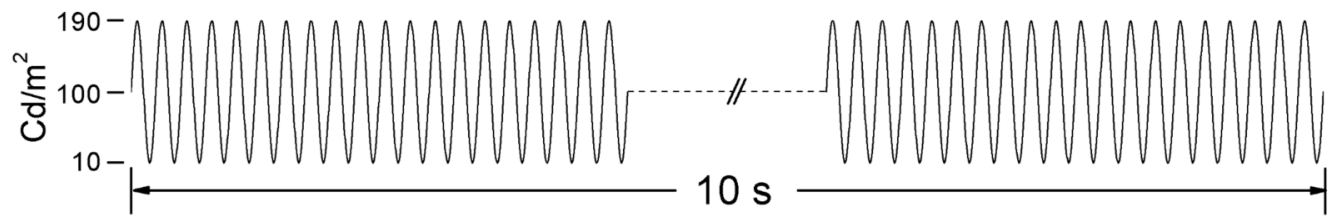

Protocol 2

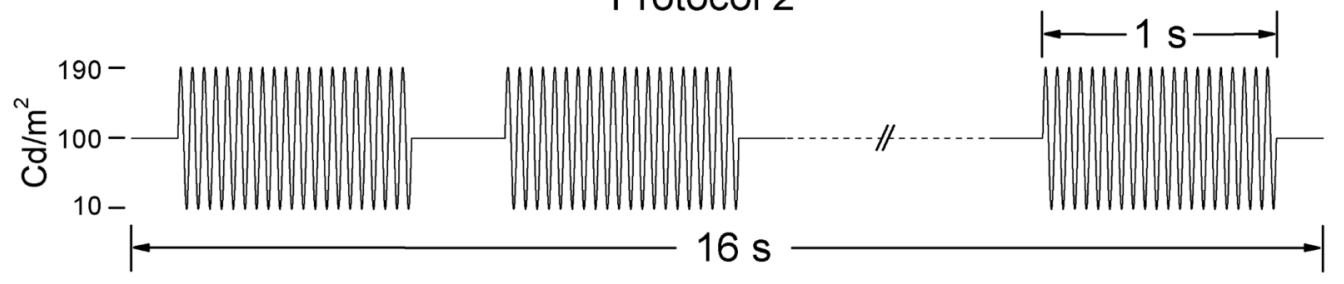

Figure 1.

Illustration of the two stimulus protocols. Protocol 1 (top) was a continuous presentation of sinusoidally modulated light with a mean luminance of $100 \mathrm{~cd} / \mathrm{m}^{2}$ and a total duration of approximately 10 seconds, containing an even number of stimulus cycles. Protocol 2 (bottom) consisted of 12 bursts of sinusoidally modulated light, each of approximately 1-s duration and containing an even number of cycles; a brief period of $0.2 \mathrm{~s}$ preceded and followed the bursts. During the interburst interval, the light level was maintained at the mean luminance of $100 \mathrm{~cd} /$ $\mathrm{m}^{2}$. The total stimulus length was approximately $16 \mathrm{~s}$. 

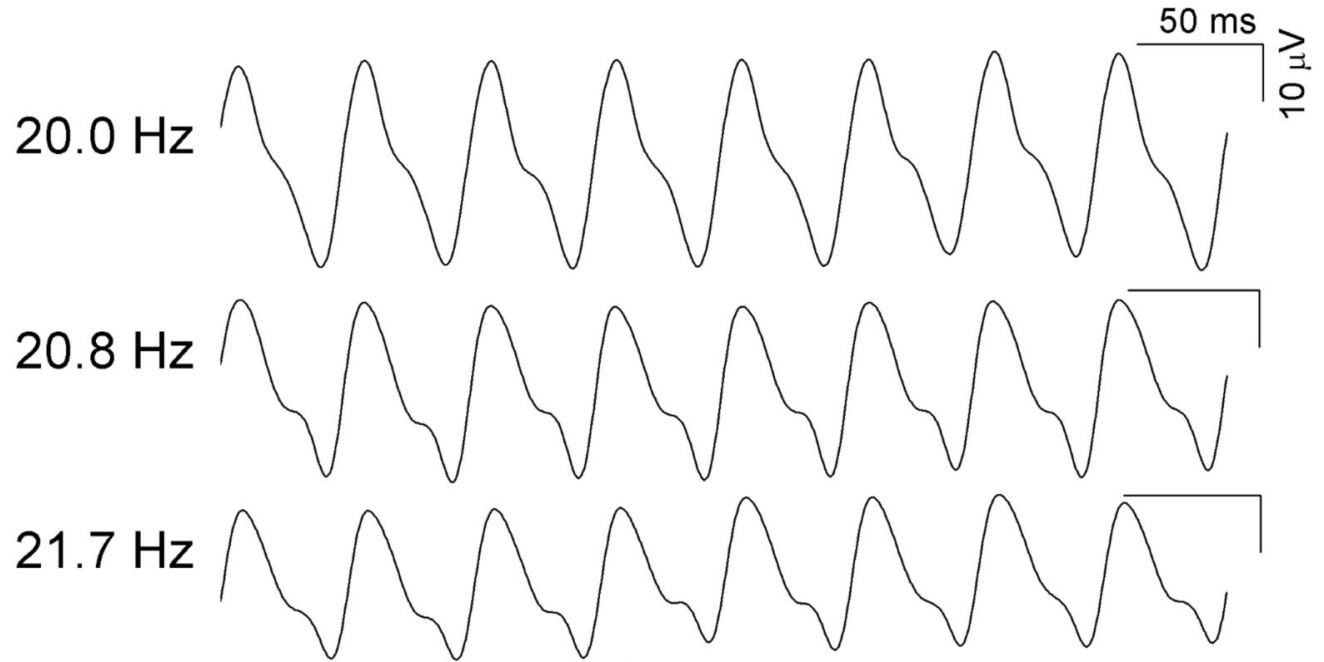

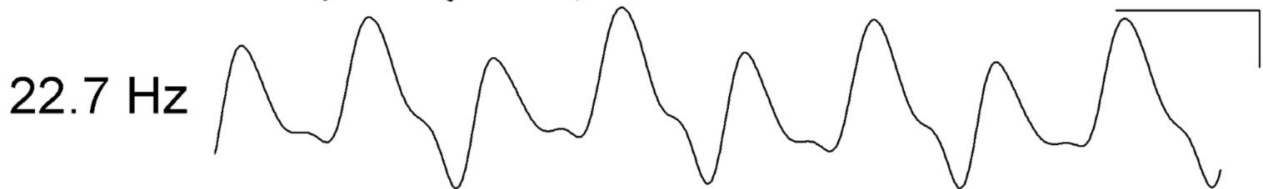
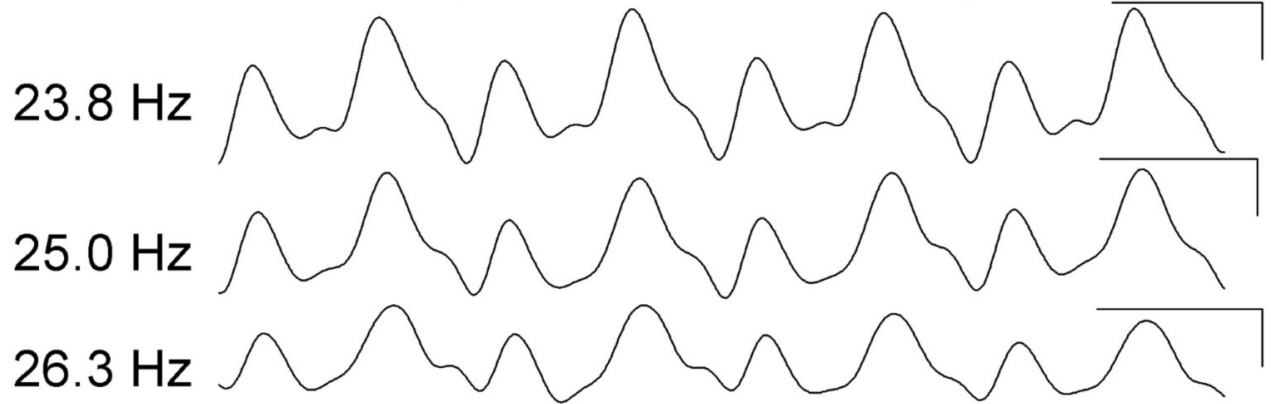

$27.8 \mathrm{~Hz}$

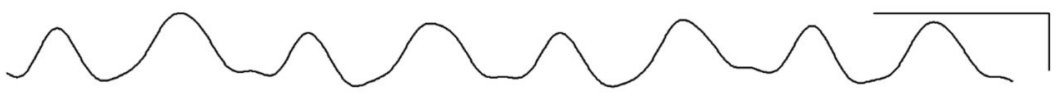

$29.4 \mathrm{~Hz}$

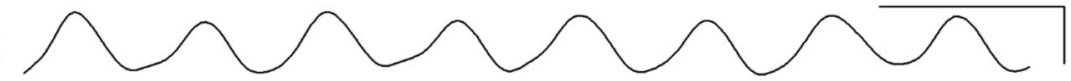

Stimulus

Figure 2.

Examples of typical ERG waveforms elicited from a rat eye using Protocol 1 at the stimulus frequencies indicated at the left. For illustrative purposes, the waveforms consist of 8-cycle segments, which were obtained by averaging successive portions of the middle $9 \mathrm{~s}$ of each recording. Responses are plotted with respect to stimulus period, with the stimulus waveform shown at the bottom; calibration bars near each waveform represent $50 \mathrm{~ms}$ and $10 \mu \mathrm{V}$, as indicated at the top of the figure. 


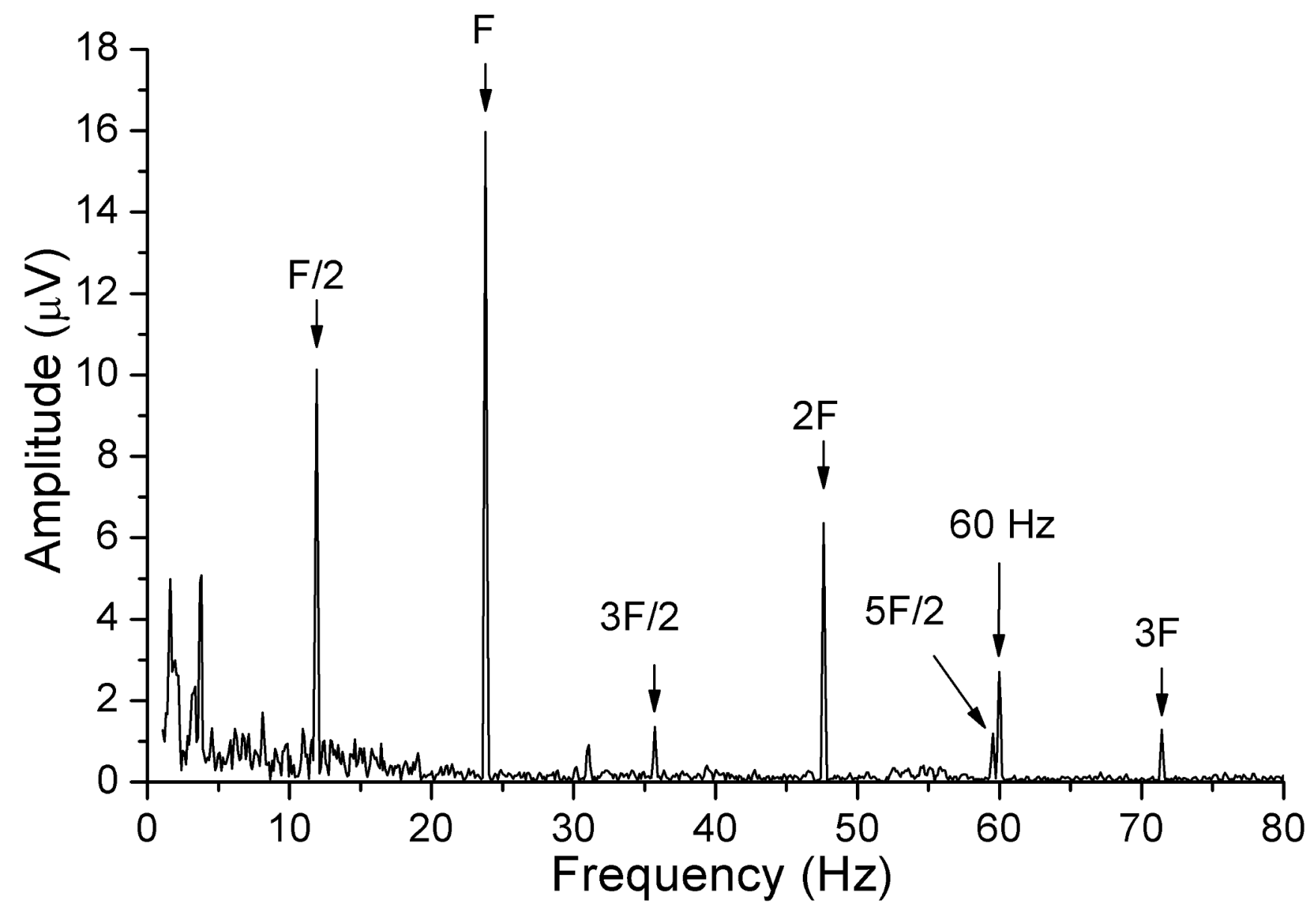

Figure 3.

Fourier spectrum of the 9-s ERG waveform at $23.8 \mathrm{~Hz}$ from the rat whose ERGs are illustrated in Fig. 2. The peaks of the various spectral components are indicated by the arrows. 

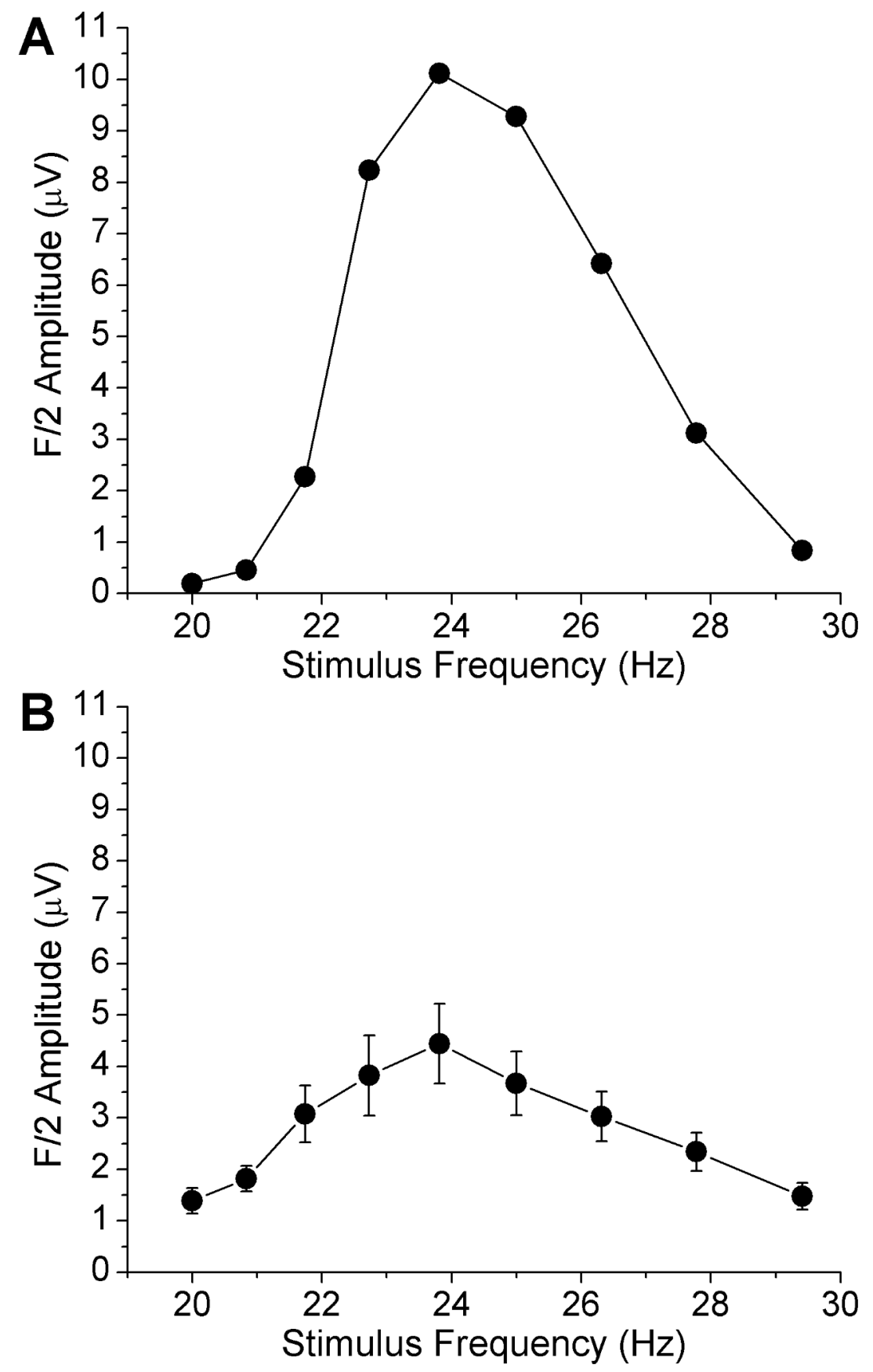

Figure 4.

A. Amplitude of the F/2 spectral component derived from the ERG waveforms shown in Fig. 2 as a function of stimulus frequency. B. Mean F/2 amplitudes of flicker ERGs obtained from 41 rat eyes as a function of stimulus frequency. Error bars represent standard errors of the means (SEMs). 


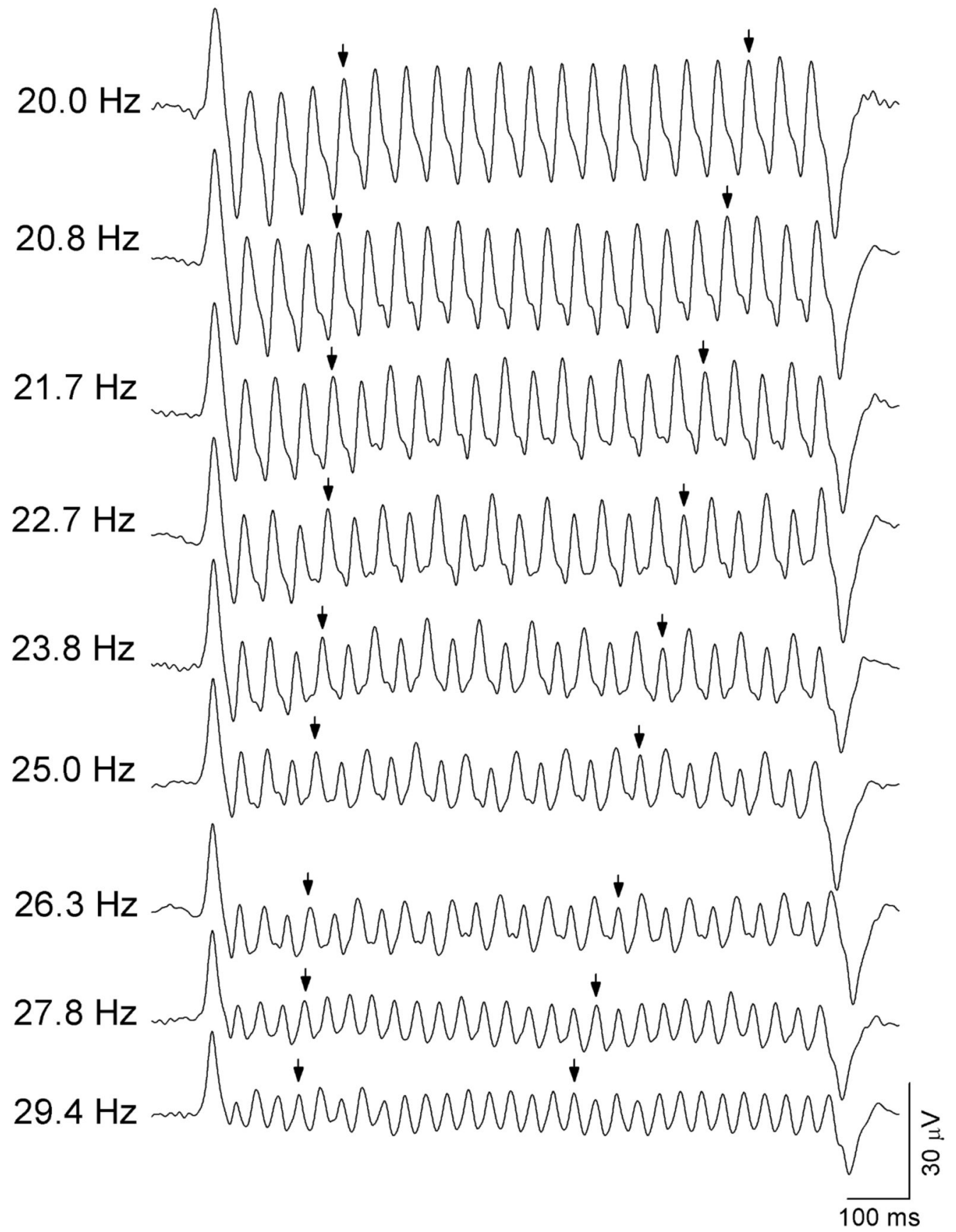

Figure 5.

Flicker ERG recordings from a typical rat eye in response to Protocol 2 at the stimulus frequencies indicated to the left of each waveform. Each trace represents the mean of the ERG responses to the 12 stimulus bursts. Arrows above each trace mark the beginning and end of the waveform segments that were used for analysis. Calibration bars are given at the bottom right. 

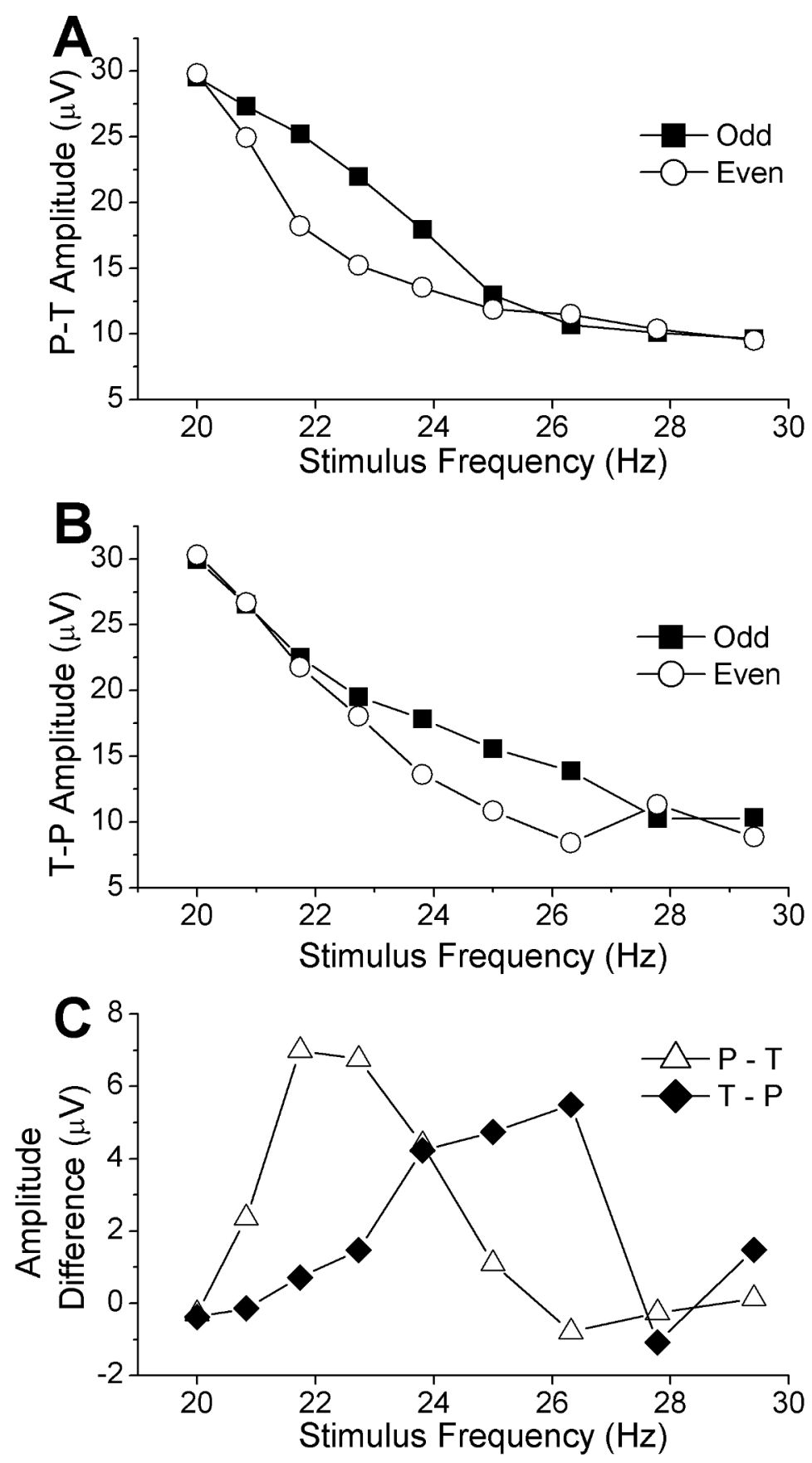

Figure 6.

Mean response amplitudes on even (circles) and odd (squares) cycles of the waveform segments indicated by the arrows in Fig. 5, using P-T (A) and T-P (B) measures. C. Differences between the amplitudes on even and odd cycles using P-T (triangles) and T-P (diamonds) measures. 


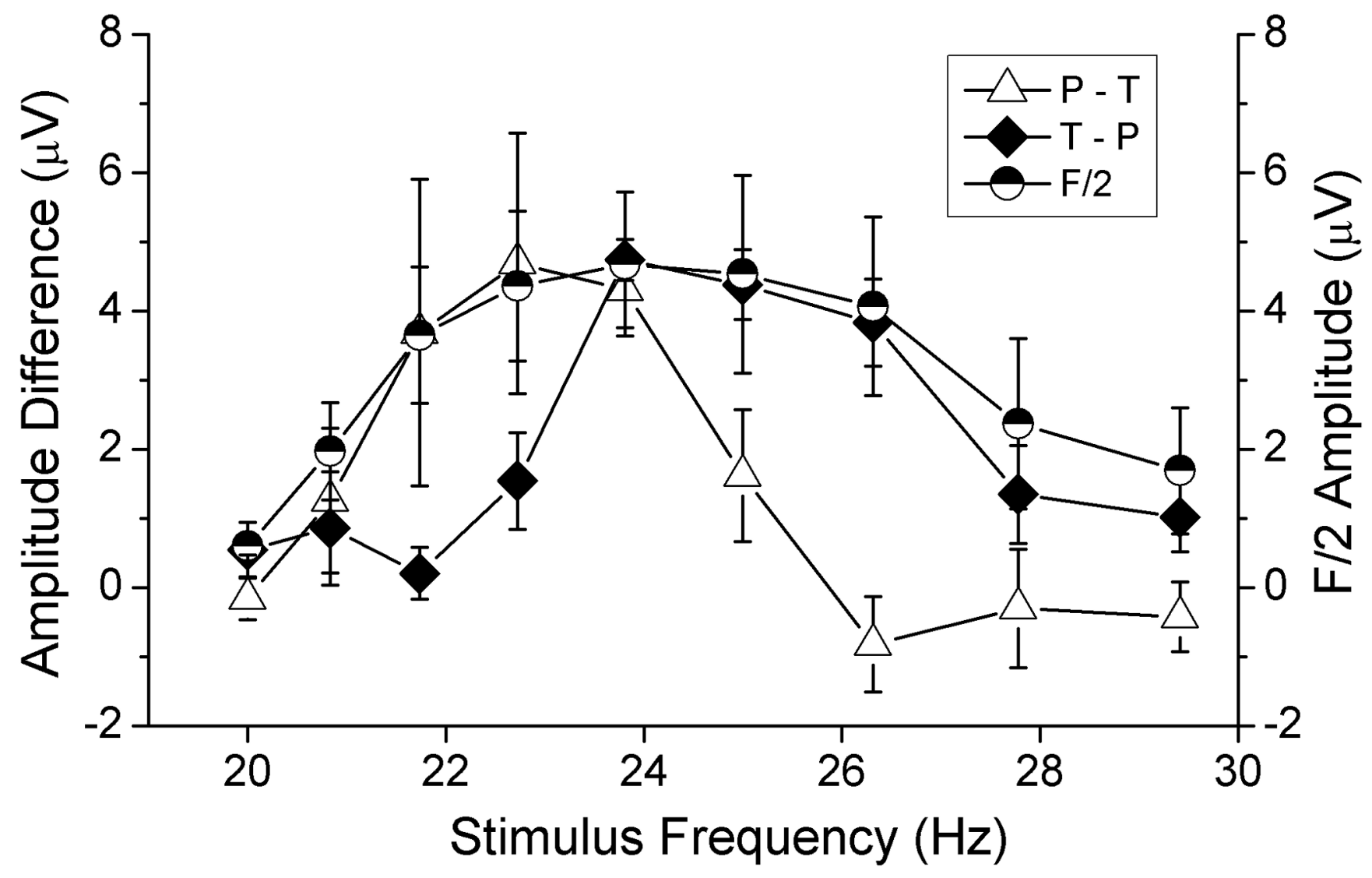

Figure 7.

Mean amplitude differences between even and odd cycles for P-T (triangles) and T-P (diamonds) measures for ERGs obtained from 8 rat eyes using Protocol 2, compared to mean $\mathrm{F} / 2$ amplitudes (half-filled circles) as derived from spectral analysis of the same ERG waveforms. Error bars represent \pm 1 SEM. 

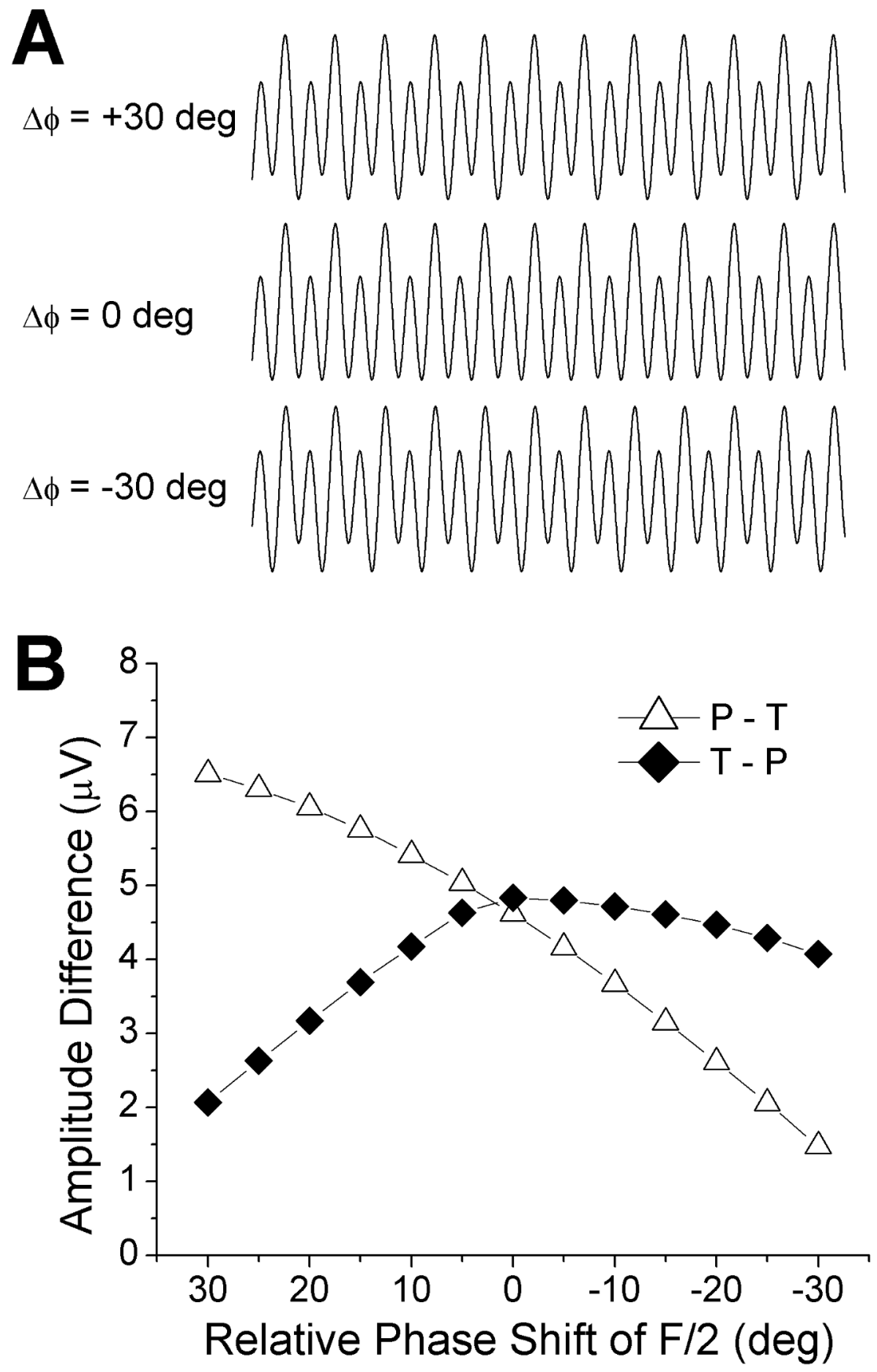

Figure 8.

A. Schematic waveforms consisting of the sum of two sine waves (F/2 and F) with amplitudes and phases derived from spectral analysis of the 23.8-Hz ERG in Fig. 5, for three relative phase offsets of F/2, where 0 represents the actual phase of F/2 in the flicker ERG. B. Amplitude difference between even and odd response cycles vs. relative phase shift of F/2 for P-T (triangles) and T-P (diamonds) amplitude measures. The $x$-axis is plotted from positive to negative because the phases of the spectral components become more negative with increasing stimulus frequency. 

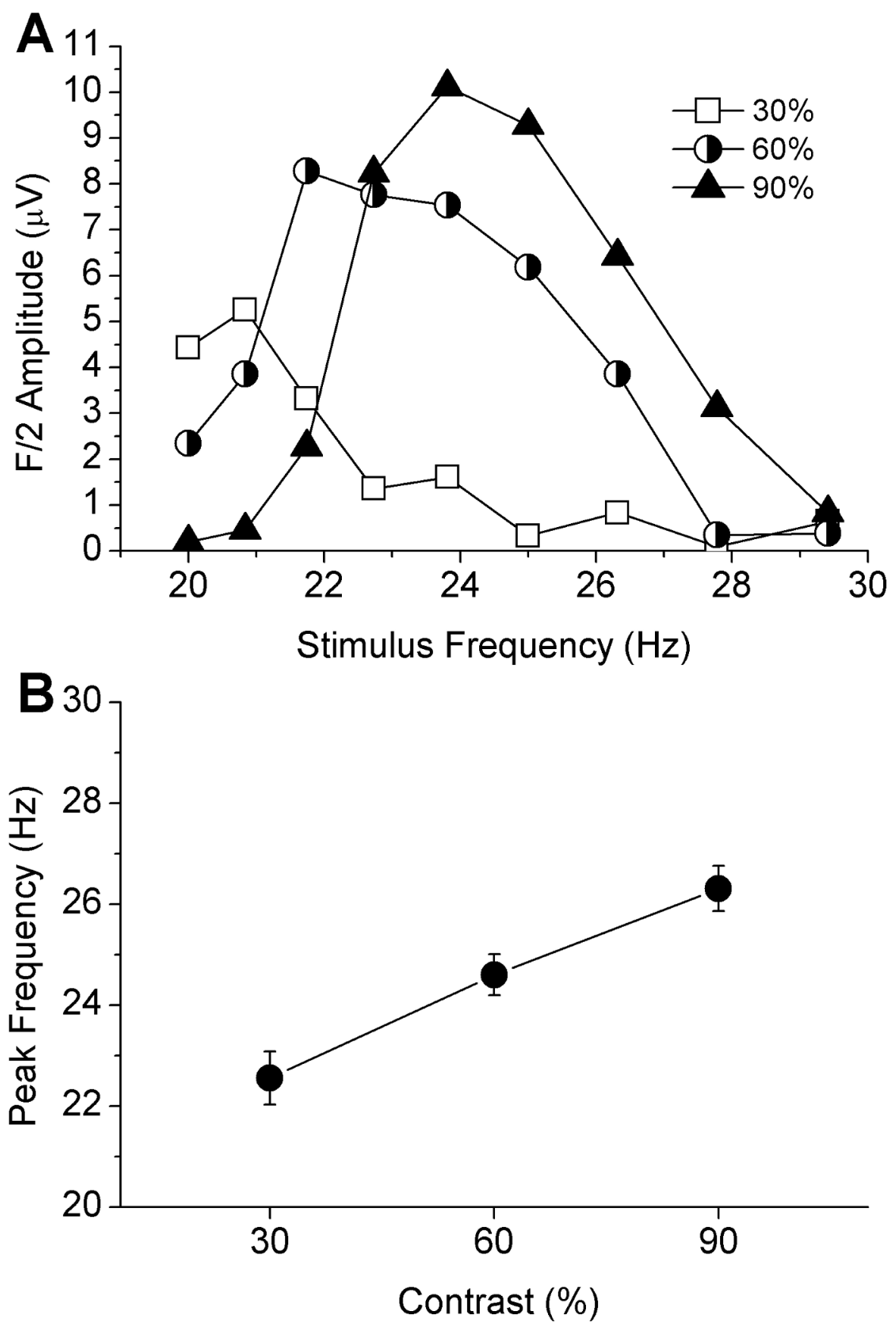

Figure 9.

A. F/2 amplitude as a function of stimulus frequency at the three contrasts indicated, as derived from spectral analysis of ERG recordings from a representative rat eye. B. Mean stimulus frequency at which the peak amplitude of period doubling occurred for ERG recordings from 9 rat eyes at three stimulus contrasts. Error bars represent \pm 1 SEM. 

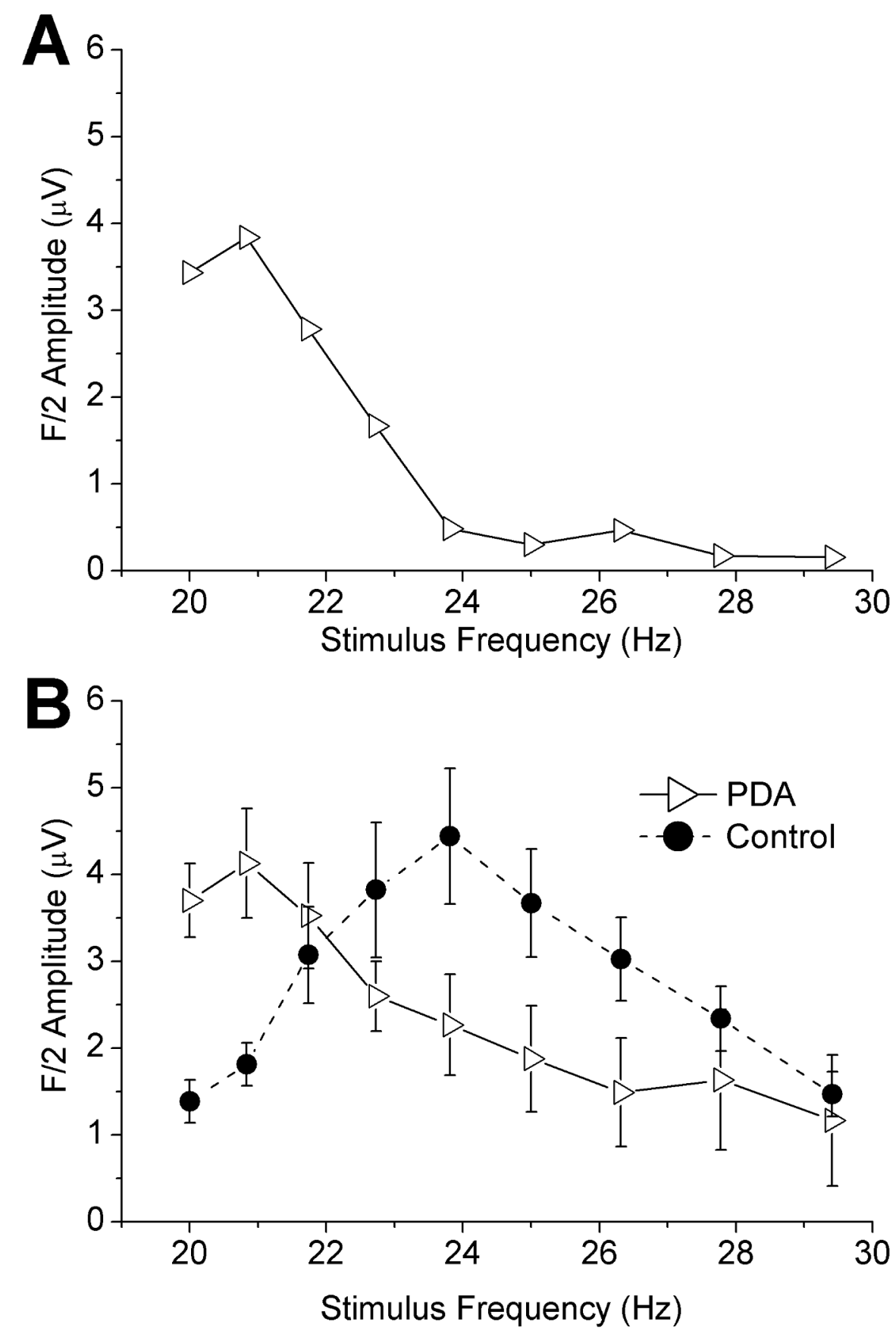

Figure 10.

A. F/2 amplitude as a function of stimulus frequency for ERGs obtained from a rat eye injected with PDA. B. Mean F/2 amplitude as a function of stimulus frequency for ERGs obtained from 12 rat eyes injected with PDA (triangles) compared to mean F/2 amplitudes obtained from 41 control eyes (circles, replotted from Fig. 4B). Error bars represent \pm 1 SEM. 